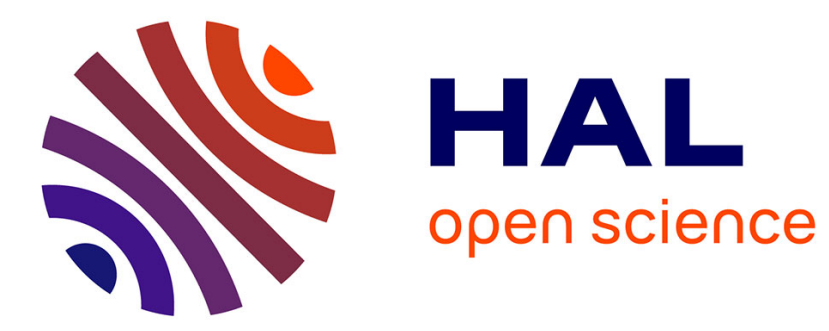

\title{
Critical exponent for 3-D percolation conductivity, revisited
}

\author{
C.D. Mitescu, M.J. Musolf
}

\section{To cite this version:}

C.D. Mitescu, M.J. Musolf. Critical exponent for 3-D percolation conductivity, revisited. Journal de Physique Lettres, 1983, 44 (16), pp.679-683. 10.1051/jphyslet:019830044016067900 . jpa-00232249

\section{HAL Id: jpa-00232249 https://hal.science/jpa-00232249}

Submitted on 1 Jan 1983

HAL is a multi-disciplinary open access archive for the deposit and dissemination of scientific research documents, whether they are published or not. The documents may come from teaching and research institutions in France or abroad, or from public or private research centers.
L'archive ouverte pluridisciplinaire HAL, est destinée au dépôt et à la diffusion de documents scientifiques de niveau recherche, publiés ou non, émanant des établissements d'enseignement et de recherche français ou étrangers, des laboratoires publics ou privés. 
Classification

Physics Abstracts

$05.60-72.60$

\title{
Critical exponent for 3-D percolation conductivity, revisited
}

\author{
C. D. Mitescu (*) and M. J. Musolf (**) \\ Department of Physics, Pomona College, Claremont, Ca. 91711, U.S.A.
}

(Reçu le 3 juin 1983, accepté le 17 juin 1983)

\begin{abstract}
Résumé. - En analysant, selon une méthode de loi d'échelle, des calculs Monte-Carlo de la conductivité électrique de réseaux cubiques simples de percolation de sites et de liens, nous obtenons l'exposant critique de la longueur de corrélation en accord avec les valeurs couramment citées, mais, pour l'exposant de la conductivité, nous trouvons $t:=2,06 \pm 0,16$ nettement plus élevé que les valeurs acceptées jusqu'ici. Ce nouveau résultat semble cependant confirmer une conjecture théorique proposée récemment.
\end{abstract}

Abstract. - When we apply finite-size-scaling analysis to Monte-Carlo calculations of the electrical conductivity of simple-cubic, bond- and site-percolation lattices, we obtain a correlation-length critical exponent in good agreement with current values but, for the conductivity exponent, we find $t=2.06 \pm 0.16$, significantly higher than the value currently cited. This new value appears, however, in essential agreement with a recently proposed theoretical conjecture.

\section{Introduction.}

Finite-size scaling methods introduced by Fisher [1] for the study of thermodynamic phase transitions have been largely applied in percolation theory to the study of the correlation-length and infinite-cluster exponents. In a recent paper [2], we have extended this method to an analysis of Monte-Carlo calculations of the electrical conductivity of percolation networks, and applied it to results obtained on 2-D lattices. This analysis allowed us to calculate the conductivity exponent $t$ as well as the correlation-length exponent from the same set of experimental data. While our results for $v$ were in good agreement with previously established values, the result we obtained for the conductivity exponent $t(=1.22 \pm 0.08)$ was significantly higher than the " best-value " $t=1.13 \pm 0.08$ generally accepted at the time our paper was submitted. Our value, consistent with the interesting theoretical conjecture subsequently proposed by Alexander and Orbach [3], was also later independently confirmed by the elegant and more precise strip-renormalization technique carried out by Derrida and Vannimenus [4], as well as in the results of Li and Strieder [5] and Lobb [6].

(*) Temporary present address : Laboratoire d'Hydrodynamique et de Mécanique Physique, E.S.P.C.I., 10 rue Vauquelin, 75231 Paris Cedex 05, France.

$(* *)$ M. J. Musolf was previously known by the name M. J. Greene. 
The results obtained in two-dimensions impelled us to apply the same technique and analysis to 3-D conductivity calculations. We have accordingly calculated, and report in this letter, the results for simple-cubic site and bond lattices. Here again, we obtain $v=0.83 \pm 0.05$ in reasonably close agreement with generally accepted $3-\mathrm{D}$ values, but we find $t / v=2.47 \pm 0.12$, -i.e., $t=2.06 \pm 0.16$ - very appreciably higher than the usually quoted value $t=1.7 \pm 0.1$ [7].

We shall briefly recall below our method for calculating the conductivity of a given lattice, the theoretical basis for the critical exponent determination, as well as the quasi-analytic interpolation technique required for the analysis of the results, all of which are described in more detail in our original paper [2]. Figure 1 displays typical conductance data $v$ s. percolation probability $p$ for lattices of various sizes, while figure 2 illustrates the determination of the critical exponents.

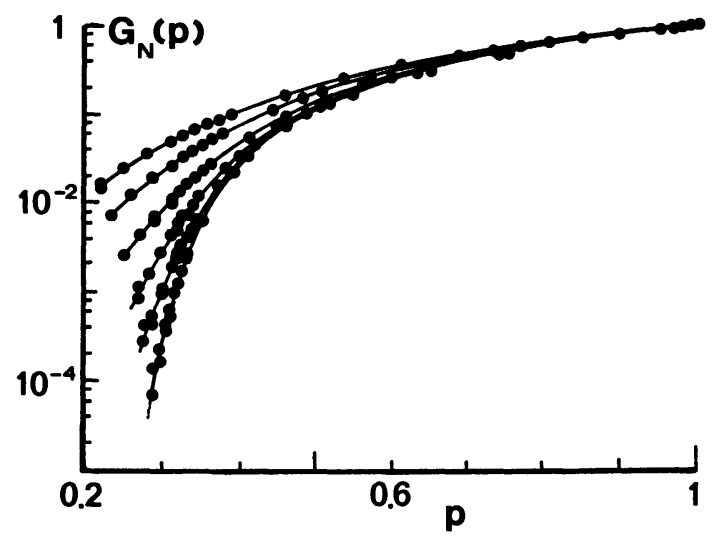

Fig. 1. - Average conductance values $G_{\mathrm{N}}(p)$ for simple-cubic site lattices, for $N=4,5,7,10,14,20$. The points represent the actual averages at various values of the percolation probability $p$ (for this lattice $p_{\mathrm{c}}=0.3115$ ). The solid lines represent the "quasi-analytic ", least-squares interpolation fit to all the data for a particular lattice size, with the interpolating function having a continuous second derivative throughout all $p$.

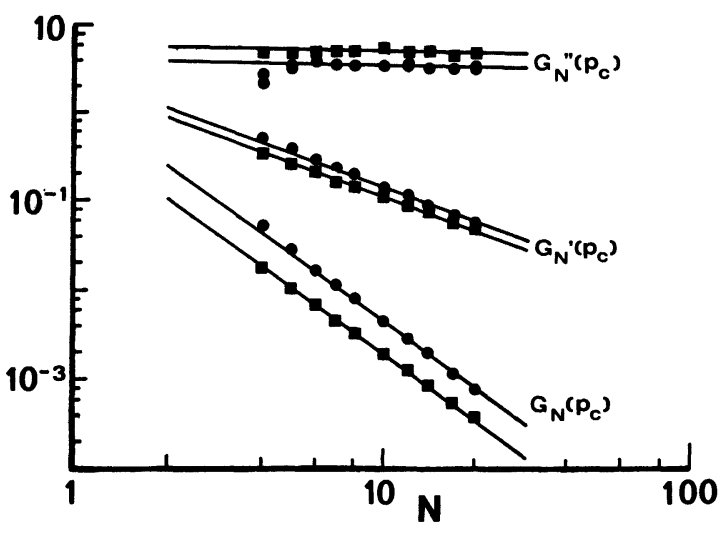

Fig. 2. - Logarithmic plot of $G_{N}\left(p_{c}\right), G_{N}^{\prime}\left(p_{c}\right), G_{N}^{\prime \prime}\left(p_{c}\right) v s$. $N$ for both the bond ( $\square$ ) and site (๑) data. The values of the points are obtained from the interpolating function. The solid lines represent the best fit for the combined data in terms of two exponents $t / v$, and $1 / v$. 


\section{Conductance calculations.}

We define, for both the bond and site case, a lattice of size $N$ to consist of $(N-1)$ horizontal planes of $N \times N$ nodes, the nodes of the uppermost plane being connected through corresponding resistors to a source of unit voltage, while those of the lowest plane are similarly connected to a ground. The conductivity, measured between the voltage source and ground, attains a maximum value of $N$ units at $p=1$, if we assume unit conductances for each lattice bond.

For a given percolation probability $p$, a given realization of the bond (or site) lattice is generated in the standard manner by a random drawing. The resultant lattice is simplified by an algorithm which eliminates all dangling, " open ", arms that contain no loops. Since this particular algorithm does not eliminate floating loops, not connected to the rest of lattice, we stabilize all such loops by assuming that every node is connected to an additional fixed $0.5 \mathrm{~V}$ source, through a conductance of $10^{-6}$ units. This does not significantly affect the potential of those nodes actually conducting, but fixes the potential of all floating loops. The lattice is then solved by an iterative over-relaxation method [8], with the overall conductance being obtained from the total energy dissipated in all conducting links, a procedure which is quadratically convergent.

We have carried out calculations for lattices sizes $N=4,5,6,7,8,10,12,14,17,20$ and for twenty-two values of the percolation probability $p$, chosen so that 12 of these are clustered near $p_{\mathrm{c}}(=0.3115$ for the site and $=0.2465$ for the bond cubic lattices $)$ and the remaining 10 spanning the complete range to $p=1$. Conductance values were thus calculated, and averaged (defining $G_{N}(p)$ for each lattice size $\left.N\right)$ over a large number of realizations at each probability $p$, the number averaged being chosen so that the total number of nodes involved was always in the range $10^{6}$ to $2 \times 10^{6}-$ e.g., for an $N=10$ lattice, having $9 \times 10 \times 10=900$ nodes, over 2000 realizations were averaged for each value of $p$. Figure 1 displays a typical set of data.

\section{Theoretical considerations.}

In order to calculate the critical exponents, we use the fact that $G_{N}(p)$, for all lattices with finite $N$, is a polynomial in $p$. At the same time, Fisher's finite-size scaling approach predicts that near $p_{\mathrm{c}}$ :

$$
G_{N}(p) \propto N^{-t / v} X(z)
$$

where $X$ is an analytic function of the variable $z=N^{1 / v} \varepsilon\left(\varepsilon=p-p_{\mathrm{c}}\right)$ behaving as $z^{t}$ for large values of $z-$ i.e., giving $G_{\infty}(p) \propto\left(p-p_{c}\right)^{t}$. $G_{N}(p)$ can therefore be expanded in a Taylor series in $\varepsilon$ about $p_{\mathrm{c}}$, yielding

$$
G_{N}(p)=N^{-t / v} X(0)+\varepsilon N^{(-t+1) / v} X^{\prime}(0)+\frac{\varepsilon^{2}}{2} N^{(-t+2) / v} X^{\prime \prime}(0) .
$$

Computation of $G_{N}\left(p_{\mathrm{c}}\right), G_{N}^{\prime}\left(p_{\mathrm{c}}\right)$ and $G_{N}^{\prime \prime}\left(p_{\mathrm{c}}\right)$ permits the extraction, from a log-log plot of these quantities against $N$, of the two critical exponents $t / v$ and $1 / v$.

\section{Interpolation method.}

The final fundamental feature of our analysis consists therefore of obtaining sufficiently accurate values, not only for $G_{N}\left(p_{\mathrm{c}}\right)$ but also for $G_{N}^{\prime}\left(p_{\mathrm{c}}\right)$ and $G_{N}^{\prime \prime}\left(p_{\mathrm{c}}\right)$, from data obtained at a finite number of values of $p$. We have achieved this by least-square fitting, over the entire range of each $G_{N}(p)$, a set of analytic polynomial arcs, requiring that not only the function value, but also its first and second derivatives, be continuous at each junction point. We find that, because the average values $G_{N}\left(p_{\mathrm{c}}\right)$ are very near zero for larger values of $N$, it is most effective to use, for this interpolation, cubic arcs, in a semilogarithmic representation of $G_{N}(p) / G_{N}(1) v s$. $p$. As a test of our interpolation technique, we have carried out an independent fit, also with cubic arcs, in a log-log representation. We find that the values of $G_{N}\left(p_{c}\right), G_{N}^{\prime}\left(p_{c}\right)$ and $G_{N}^{\prime \prime}\left(p_{c}\right)$ computed by the two methods, 
are in excellent agreement. (We might also point out that, since the values of the above functions at $p_{\mathrm{c}}$ are obtained from the interpolation function, which gives equal weight to all data points, we feel our results should be relatively free of effects due to any slower convergence at $p_{c}$ itself).

The solid lines on figure 1 present a typical set of interpolation curves together with the underlying Monte-Carlo data.

\section{Results.}

Figure 2 displays a log-log plot of $G_{N}\left(p_{c}\right), G_{N}^{\prime}\left(p_{c}\right)$, and $G_{N}^{\prime \prime}\left(p_{c}\right) v s . N$ for both the bond and site lattices. The straight lines through the data correspond to a least-squares fit through the combined bond and site data, in terms of the two power-law exponents

$$
\text { i.e., } \quad t=2.06 \pm 0.16 \quad v=0.83 \pm 0.05 \text {. }
$$

Independent analysis of the data for the two different types of lattices gives, for the bond problem,

i.e.,

$$
\begin{array}{rlrl}
t / v & =2.36 \pm 0.08 & 1 / v & =1.17 \pm 0.05 \\
t & =2.02 \pm 0.12 & v & =0.85 \pm 0.03
\end{array}
$$

while, in the site case,

i.e.,

$$
\begin{array}{rlrl}
t / v & =2.58 \pm 0.10 & 1 / v & =1.24 \pm 0.06 \\
t & =2.08 \pm 0.14 & v & =0.81 \pm 0.04
\end{array}
$$

We observe therefore that, within experimental accuracy, the bond and site data are consistent with the expectations of universality for the critical exponents.

The overall value $v=0.83 \pm 0.05$ is quite consistent with currently cited values $(v=0.85-$ 0.88 ) [9]. Our value of $t / v=2.47 \pm 0.12$ (i.e., $t=2.06 \pm 0.16$ ) is new and, as stated above, significantly higher than the $t=1.7 \pm 0.1$ [7] commonly accepted heretofore.

We seem to be in a situation where the value of $t$ has undergone, with the years, a certain amount of gyration. From one of the earliest values of 2 [10], it has appeared to stabilize around $t=1.7$, though with an occasional fluctuation [11] $(t=1.85 \pm 0.10)$. We are at this stage in a period where this particular value appears to « destabilize » both from experimental and theoretical considerations.

On the experimental side, apart from this work, independent random-walk data of Mitescu and Roussenq [12] give a diffusion time-scale exponent $\theta(=t+2 v-\beta)=3.35 \pm 0.10$ (whence $t=2.06$ ) very consistent with our results here. On the other hand, Havlin et al. [13], also from diffusion data, seem to drift in the opposite direction with $t=1.5 \pm 0.1$.

On the theoretical side, Skal-Shklovskii-de Gennes [14, 15] superlattice arguments had predicted an expression for the conductivity exponent

$$
t=(d-2)+\zeta
$$

with the exponent $\zeta$, originally suggested to be exactly 1 by de Gennes, being at least close to unity.

More recently, Alexander and Orbach [3] have introduced a spectral dimensionality (governing the scaling of vibrational excitations, "fractons " on a self-similar lattice) which they define as $\overline{\bar{d}}=\frac{2 \bar{d} v}{\theta}=\frac{2(d v-\beta)}{(t+2 v-\beta)}$ where $\bar{d}(=d-\beta / v)$ is the fractal dimensionality of a percolation system at the critical point, $d$ is the Euclidian dimension, and $\theta$ has been defined just above. 
Conjecturing that $\overline{\bar{d}}$ has the universal value $4 / 3$ in all dimensions, they then obtain

$$
t=[(3 d-4) v-\beta] / 2 .
$$

Using $\beta / v=0.50$ [16], $v=0.86$, this predicts $t=1.94$, or $t / v=2.25$. (We might remark in passing that the expressions of Skal-Shklovskii-de Gennes and Alexander-Orbach are mutually consistent if $\zeta=\bar{d} v / 2$ ).

We consider that our data are consistent with the Alexander-Orbach conjecture, but appear to exclude the earlier value $t=1.7$. We must point out however, that the error estimates in our results are based on the internal consistency of our data, and do not include possible systematic errors that might result from corrections to scaling. The agreement in the value of $v$, however, inclines us to have a certain amount of confidence in the value of $t$ as well.

After the results presented in this letter had been cited in the review of Mitescu and Roussenq [12], calculations were begun, using different numerical techniques, by Derrida et al. [17], and also by Pandey and Stauffer [18]. We are informed that these also appear to confirm a value of $t$ close to 2, and good agreement with the Alexander-Orbach conjecture.

\section{Acknowledgments.}

We have appreciated useful discussions with E. Guyon and D. Stauffer. One of us (M. J. M.) was the holder of a Shell Foundation undergraduate research grant at Pomona College in the summer of 1982.

\section{References}

[1] Fisher, M. E., Critical Phenomena : Proc. Enrico Fermi Internat. Phys. Summer School (Course LI), Ed. : M. S. Green (Academic Press : NY), 1971, p. 1-99.

[2] Mitescu, C. D., Allain, M., Guyon, E. and Clere, J. P., J. Phys. A 15 (1982) 2523.

[3] Alexander, S. and Orbach, R., J. Physique Lett. 43 (1982) L-625.

[4] Derrida, B. and Vannimenus, J., J. Phys. A 15 (1982) L-557.

[5] Li, P. S. and Strieder, W., J. Phys. C 15 (1982) 6591 and L-1235.

[6] LOBB, C. J., private communication to D. Stauffer.

[7] Kirkpatrick, S., I11 Condensed Matter, Edited by R. Balian, R. Maynard and G. Toulouse (North Holland, Amsterdam) 1979, p. 231.

[8] Webman, I., Jortner, J. and Cohen, M., Phys. Rev. B 11 (1975) 2885.

[9] Harris, A. B., Phys. Rev., to be published.

[10] Adler, D., Flora, L. P. and Senturia, S. D., Solid State Commun. 12 (1973) 9.

[11] Clerc, J. P., Giraud, G., Alexander, S., Guyon, E., Phys. Rev. B 22 (1980) 2489.

[12] Mitescu, C. D. and RoussenQ, J., Ann. Israel Phys. Soc. 5 (1983) 81.

[13] Havlin, S., Ben-Avraham, D. and Sompolinsky, H., Phys. Rev. A (1983) to be published.

[14] Skal, A. S. and Shklovskit, B. I., Fiz. Fekh. Poluprovodn. 8 (1974) 1582, Sov. Phys. Semicond. 8 (1975) 1029.

[15] De Gennes, P. G., J. Physique Lett. 37 (1976) L-1.

[16] Margolina, A., Herrmann, H. J. and Stauffer, D., Phys. Lett. 69A (1982) 73.

[17] Derrida, B., Stauffer, D., Herrmann, H. J., Vannimenus, J., to be published.

[18] Pandey, R. B., Stauffer, D., to be published. 